\title{
Presynaptic Long-Term Facilitation at the Crayfish Neuromuscular Junction: Voltage-Dependent and lon-Dependent Phases
}

\author{
J. M. Wojtowicz and H. L. Atwood \\ Department of Physiology, University of Toronto, Toronto M5S 1A8, Ontario, Canada
}

Long-term facilitation (LTF) of synaptic transmission was investigated in the crayfish opener muscle to determine the factors necessary for its induction and expression. LTF was induced without action potentials by intracellular depolarization of presynaptic nerve terminals. Following induction, the synaptic transmission was enhanced by about $80 \%$ for a period of several hours. Intracellular recordings from preand postsynaptic cells, combined with ionic and pharmacological tests, permitted dissection of LTF into 2 phases: an initial tetanic phase that depended on the presence of both sodium and calcium lons and a subsequent long-lasting phase. This latter long-lasting enhancement of synaptic transmission was induced by repeated depolarizations of synaptic terminals but did not depend on the influx of sodium or calcium ions or on intracellular release of calcium ions. Both tetanic and long-lasting phases of LTF are attributable to activity of a single neuron, i.e., they are homosynaptic phenomena. Furthermore, LTF is associated with an increase of quantal release, whereas the size of quanta remains unchanged. During the long-lasting phase of LTF, the nerve terminal releases more transmitter for a given depolarization than before induction of LTF. Thus, the locus of LTF is presynaptic. Our findings suggest the presence of a voltage-dependent mechanism in the presynaptic membrane different trom voltage-gating of $\mathrm{Na}$ or $\mathrm{Ca}$ channels. Such a mechanism may be important in the establishment of long-lasting synaptic changes at the crayfish neuromuscular junction and perhaps in other neural systems.

Synaptic transmission is dependent upon influx of extracellular calcium ions $\left(\mathrm{Ca}^{2+}\right)$ into the presynaptic terminals of transmitting nerve cells (Katz and Miledi, 1967; Augustine et al., 1987). Various forms of synaptic plasticity-including short-term facilitation (Katz and Miledi, 1968), augmentation (Erulkar, 1983), posttetanic potentiation (Rahamimoffet al., 1980; Erulkar, 1983), long-tcrm facilitation (Atwood and Wojtowicz, 1986), and longterm potentiation (Lynch et al., 1983)-have been linked to entry and accumulation of $\mathrm{Ca}^{2+}$ and/or $\mathrm{Na}^{+}$during neural activity. Although a voltage-dependent step for transmitter release

\footnotetext{
Received Jan. 19, 1988; revised Mar. 31, 1988; accepted Apr. 29, 1988.

The research was supported by a grant from the Medical Research Council of Canada. We are grateful to Marianne Hegstrom-Wojtowicz and Alma Cull for assistance with the manuscript preparation. We also thank Drs. M. Charlton, U. De Boni, I. Mercier, P. Pennefather, and D. van der Kooy for reading and commenting on an earlier version of the manuscript.

Correspondence should be addressed to Dr. J. M. Wojtowicz, Department of Physiology, Medical Sciences Building, University of Toronto, Toronto M5S 1A8, Ontario, Canada.

Copyright (C) 1988 Society for Neuroscience $0270-6474 / 88 / 124667-08 \$ 02.00 / 0$
}

has been proposed for crayfish and frog neuromuscular junctions (Dudel et al., 1983; Dudel, 1984), evidence for this proposal is disputed (Atwood and Wojtowicz, 1986; Parnas and Parnas, 1986; Zucker and Lando, 1986), and the hypothesis is still under investigation. Recent evidence suggests voltage-dependent, calcium-independent transmitter release at retinal synapses (Schwartz, 1987). Until recently there has been no evidence for voltage-dependent induction of long-term synaptic plasticity; however, recent evidence suggests that calcium-independent, depolarization-dependent long-term potentiation can occur in cerlain neural circuits of the mammalian hippocampus (May et al., 1987). We report here evidence for voltage-dependent longterm enhancement of transmission at crayfish neuromuscular synapses. These synapses resemble central synapses in many aspects of their physiology (Atwood, 1976; Atwood and Wojtowicz, 1986). This novel voltage-dependent homosynaptic plasticity opens up another class of synaptic changes that could be important in altering performance in both CNS and PNS.

Long-term facilitation (LTF), first described at crustacean neuromuscular junctions by Sherman and Atwood (1971), consists of progressive growth in amplitude of evoked excitatory postsynaptic potentials (EPSPs) during stimulation of a motor axon at frequencies above $10 \mathrm{~Hz}$. After stimulation has been stopped, the facilitated state decays relatively rapidly for the first 10-20 min but thereafter much more slowly; persistent enhancement can last for several hours to at least a day (Lnenicka and Atwood, 1985). It was proposed originally that induction of LTF is primarily sodium dependent, with a secondary increase of intracellular calcium being responsible for the enhanced probability of transmitter release (Atwood et al., 1975). We now have evidence that while both calcium and sodium participate in the early phases of LTF, long-lasting enhancement of transmitter release is produced by a voltage-dependent mechanism rather than by ionic fluxes.

\section{Materials and Methods}

Experiments were performed on 32 small crayfish (Procambarus clarkii) obtained from Atchafalaya Biological Supplies Company in Louisiana. We used the dissecting and recording techniques described in detail in previous publications (Wojtowicz and $\Lambda$ twood, 1984, 1985a, 1986). Briefly, the crayfish opener neuromuscular junction preparation (from the first walking leg) was set up in vitro for simultaneous intracellular recordings from presynaptic nerve terminals and postsynaptic muscle cells before, during and after induction of LTF. Ionic composition of the extracellular medium could be varied within 2-3 min, and pharmacological agents could be applied in known concentrations. In the present experiments, LTF was induced by depolarizing pulses applied to the nerve terminal through an intracellular microelectrode. This permitted experiments to be done in modified perfusion solutions, in which action potentials were abolished and various channel blockers em- 
Figure 1. A, Examples of depolarizing current pulses applied to the presynaptic nerve terminal and the resulting voltage deflections. Pulses were passed via an intracellular microelectrode using a bridge circuit. The resting membrane potential was $-70 \mathrm{mV}$. Arrowheads indicate the beginning and the end of the pulses. $B$, Depolarizing responses (EPSPs) in a muscle cell corresponding to 3 levels of presynaptic depolarization. Each response is an average corresponding to 30 stimuli applied at $1 \mathrm{~Hz}$. EPSPs are preceded by artifacts produced by electrical coupling between pre- and postsynaptic microelectrodes. Resting membrane potential was $-68 \mathrm{mV}$. $C$, Transfer curves showing the relationship between the presynaptic current and postsynaptic responses on linear (left) and logarithmic (right) axis. Each point corresponds to the amplitude of averaged EPSPs such as shown in $B$. The curves provide a comparison of the functional state of nerve terminals before and after induction of long-lasting phase of LTF. It is evident from these data that there was more transmitter release during LTF for the same amount of current. The initial part of the logarithmic plot was fitted by eye to a straight line yielding a slope of 4.0. Characteristically, the slope of such lines did not change during LTF.
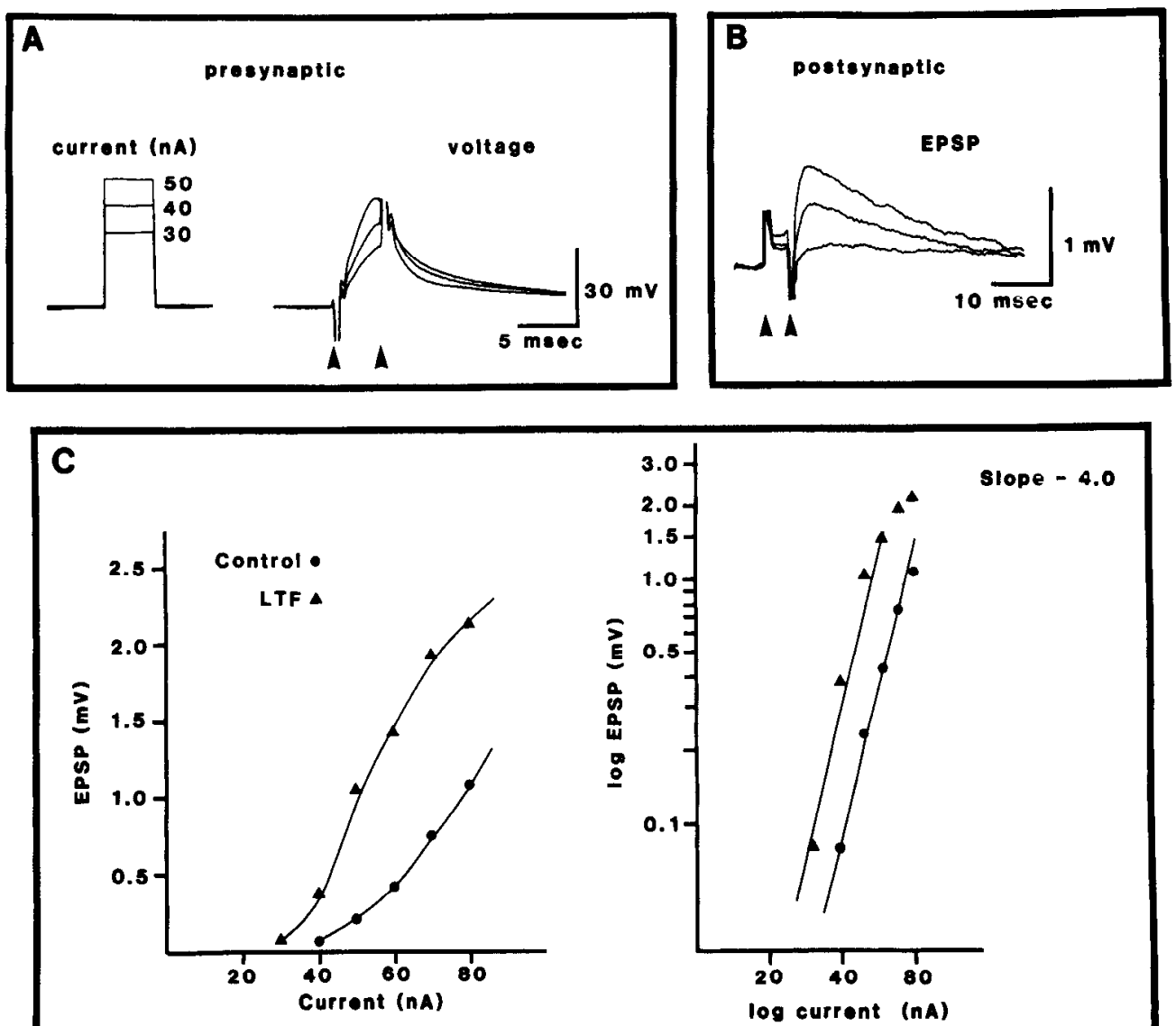

ployed. Temperature of the perfusion solutions was kept at $13^{\circ} \mathrm{C}$ in all experiments.

Samples of the pre- and postsynaptic records are shown in Figure 1. The presynaptic panel (Fig. $1 A$ ) shows that in a preparation treated with $0.1 \mu \mathrm{M}$ TTX graded constant-current pulses produce corresponding graded voltage responses in the presynaptic tcrminal at the site of current injection. Typically we injected currents ranging between 30 and $80 \mathrm{nA}$ by means of a bridge circuit (Dagan 8500 amplifier) and standard glass microelectrodes filled with $3 \mathrm{M} \mathrm{KCI}$ (resistances 8-10 M $\Omega$ ). The duration of current pulses was 3-5 msec. The postsynaptic panel (Fig. $1 B$ ) illustrates the depolarizing synaptic responses (EPSPs) obtained in a muscle fiber during repeated presynaptic stimulation at a frequency of $1 \mathrm{~Hz}$. The current pulses were considerably longer than action potentials, which are usually $1-1.5 \mathrm{msec}$ at half-width in this preparation (Wojtowicz and Atwood, 1984). Shorter pulses did not produce transmitter release presumably because the spatial decay of voltage from the site of current injection to the site of transmitter release was excessive. Consequently, the depolarizations experienced by the release sites were probably smaller but more prolonged than with action potentials.

The data from several experiments were plottcd to obtain transfer curves, which could be displayed on either linear or logarithmic axes (Fig. 1C). In the graphs we plotted the current instead of the presynaptic voltage on the abscissa because at large currents a proper balance of the presynaptic electrode and therefore a proper measurement of the voltage could not be assured due to an increase in electrode resistance. Moreover, the precise value of the voltage deflection at the exact site of release of neurotransmitter could not be known with certainty (see Appendix in Wojtowicz and Atwood, 1984, for a discussion of this problem). Nevertheless, for currents below $60 \mathrm{nA}$ a reasonable measurement of membrane potential could be made and therefore the technique was useful in comparing the synaptic properties before and after the establishment of LTF, as well as for the induction of LTF.

The composition of the standard (van Harreveld's) perfusing solution was as follows: $206 \mathrm{~mm} \mathrm{NaCl}, 5.3 \mathrm{~mm} \mathrm{KCl}, 13.5 \mathrm{mM} \mathrm{CaCl}, 2.5 \mathrm{~mm}$ $\mathrm{MgCl}_{2}$, and $1 \mathrm{mM}$ HEPES buffer adjusted to $\mathrm{pH} 7.4$. In calcium-free solutions $\mathrm{CaCl}_{2}$ was replaced by $13.5-30 \mathrm{mM} \mathrm{MgCl}$, by $6 \mathrm{mM} \mathrm{MnCl}_{2}$, by $0.5-1.0 \mathrm{mM} \mathrm{NiCl}_{2}$, or by $0.1-0.2 \mathrm{mM} \mathrm{CoCl}_{2}$. In some cases, $1 \mathrm{~mm}$ EGTA was added to the calcium-free solutions. In low-sodium solutions the concentration of $\mathrm{NaCl}$ was lowered to $20 \mathrm{~mm}$ and the balance substituted with either choline chloride or $N$-methyl-D-glucamine.

In some experiments the calcium buffer BAPTA-AM (Molecular Probes) was used to reduce the activity of calcium ions in the presynaptic terminals during induction of LTF. BAPTA-AM is an acetoxymethyl tetraester of BAPTA (Tsien, 1980, 1981). BAPTA-AM is membrane permeable and once inside the cell is expected to be esterified and accumulated in concentrations considerably higher than in the extracellular space. At the beginning of each such experiment we applied 25 $\mu \mathrm{M}$ BAPTA-AM to the perfusion solution for about $1 \mathrm{hr}$. This dose was sufficient to reduce the release of transmitter to $44.7 \%$ when the presynaptic terminal was stimulated at $5 \mathrm{~Hz}$ during the pretest period preceding the induction of LTF. Since it is clear that at $5 \mathrm{~Hz}$ the evoked EPSPs undergo short-term facilitation by about 8 -fold (see Results), there is likely to be accumulation of residual calcium in the terminals and a consequent increase in the calcium concentration by about $70 \%$ with respect to the resting level (assuming cooperativity value between calcium and the release process of 4 , see Atwood and Wojtowicz, 1986). BAPTA probably buffered this residual calcium, as well as the transients of calcium due to repeated presynaptic depolarizations, and therefore reduced the amplitude of EPSPs. In Ca-free, Mn-containing medium (e.g., Fig. 5), there were no calcium transients and no visible calcium accumulation, so all buffering capacity of BAPTA was left to handle a possible, albeit unlikely, release of calcium from intracellular stores. Therefore, we infer that in such conditions the dose of BAPTA was sufficient to maintain the intracellular calcium concentration at a level substantially lower than during $5 \mathrm{~Hz}$ stimulation and possibly lower than at resting level.

\section{Results}

\section{LTF with and without action potentials}

The time course of LTF produced by action potentials evoked with stimulation of the motor axon is shown in Figure $2 A$, which 
serves as a comparison for pulse-evoked LTF illustrated in Figures $2 B, 3$, and 5 . In an earlier report (Wojtowicz and Atwood, 1985a), we divided LTF into 4 components: the tetanic phase and 3 decay phases. However, to simplify the presentation and discussion of the results of this study, we shall consider LTF to comprise 2 components. The first component of LTF (designated "Tetanic phase" in Fig. $2 A$ ) includes the period of stimulation and the period of rapid decay of EPSPs lasting approximately 15-20 $\mathrm{min}$. The second component (designated "Longlasting phase") includes the prolonged enhancement of synaptic transmission lasting several hours in vitro and up to one day in vivo. The tetanic phase serves to induce the long-lasting phase (LLP).

In preparations in which the presynaptic action potential was abolished by $0.1 \mu \mathrm{M}$ TTX, repeated application of standard depolarizing pulses 30-60 nA in amplitude and 3-5 msec in duration at $5 \mathrm{~Hz}$ produced substantial transmitter release. We used $1 \mathrm{~min}$ bouts of such stimulation to establish a baseline during the pretest period (Fig. $2 B$ ). The amplitude of the pulses was carefully adjusted to produce EPSPs of the same magnitude as those produced by action potentials prior to the application of TTX and potassium channel blockers. Subsequently, the injected current was kept constant for the remainder of the experiment.

Stimulation of the terminal at $20 \mathrm{~Hz}$ for $10 \mathrm{~min}$ reliably produced clear LTF. Both the tetanic and long-lasting phases were present, as can be seen by comparing the pretest, tetanic, and the recovery records in Figure $2 B$. Characteristically, there was no consistent changes in the amplitude of the presynaptic voltage deflection produced by the current pulse, indicating no change in the membrane resistance.

\section{Ionic dependence of LTF}

We performed experiments to determine the ionic dependence of 2 phases of LTF (Fig. 3). Sodium dependence was assessed by stimulating the nerve terminal by local current pulses in the presence of $0.1 \mu \mathrm{M}$ TTX, as described above. Under such conditions the influx of sodium ions, normally brought about by action potentials, was prevented. The preterminal action potential is known to be mainly sodium dependent (Wojtowicz and Atwood, 1984). The tetanic (growth) phase of LTF was truncated and the initial decay phase was more rapid, while LLP was fully expressed (Fig. $3 A$ ). The remaining core of the tetanic phase is likely to be a result of calcium accumulation in the presynaptic terminal according to the residual calcium theory (reviewed in Atwood and Wojtowicz, 1986). Supplementary experiments in which $90 \%$ of sodium ions were replaced with either choline or $N$-methyl-D-glucamine together with the addition of TTX yiclded similar results. In 3 such experiments the average enhancement of EPSP recorded $30 \mathrm{~min}$ after the tetanus was $62 \%$ (range, $20-91 ; \mathrm{SD}=37$ ). This value does not appear to be significantly lower than $81 \%$ (range, 30-117; SD $=33 ; n=5$ ) enhancement recorded in the presence of normal sodium concentration with or without TTX (see Fig. 3A and Fig. 4 in Wojtowicz and Atwood, 1986). These experiments suggest that sodium influx is not necessary for the induction of LLP. However, the present results together with the evidence presented in earlier studies (Wojtowicz and Atwood, 1985a; Atwood and Wojtowicz, 1986) make a strong case for a role of sodium during the tetanic phase.

Possible involvement of potassium (K) channels in LTF was assessed since other neural systems show plasticity that depends

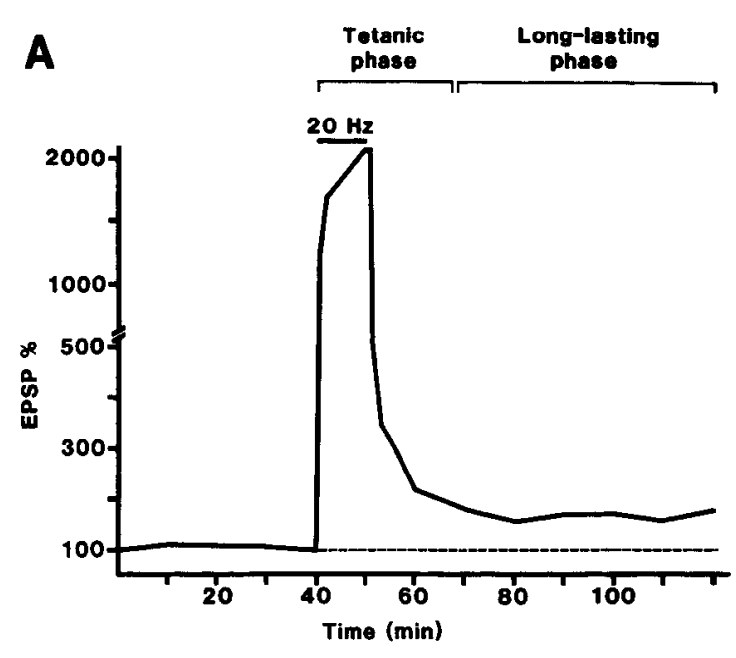

B
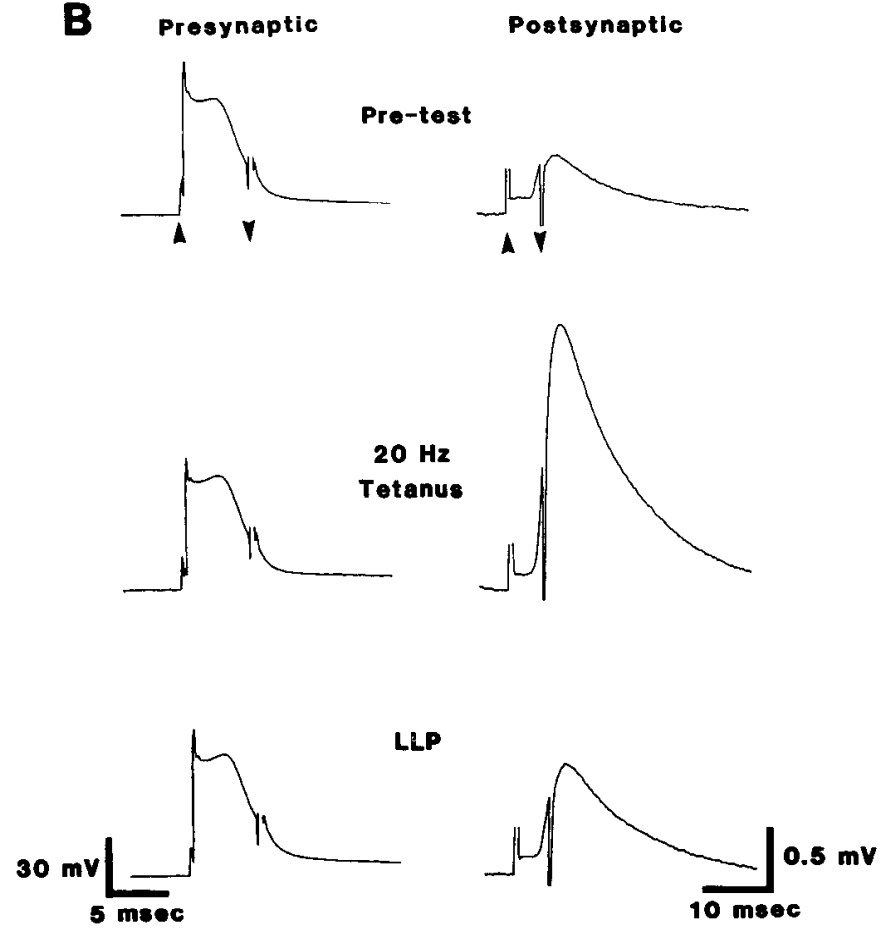

Figure 2. A, Time course of LTF (modified from Wojtowicz and Atwood, 1985) shows a pretest period, during which evoked EPSPs are measured during $5 \mathrm{~Hz}$ stimulation once every $10 \mathrm{~min}$; a tetanic phase, during which induction of LTF takes place; and long-lasting phase of LTF. In the present context, the time course of such action potentialinduced LTF is given as the basis of comparison with LTF produced by local depolarizing pulses illustrated in subsequent figures. $B$. Demonstration of LTF produced by local depolarizing pulses applied to the axon terminal by intracellular stimulation. Depolarizing pulses, $60 \mathrm{nA}$, were applied (presynaptic) and the resulting EPSPs recorded in a muscle cell (postsynaptic). Arrows indicate current pulse artifacts. Stimulation of the terminal at $20 \mathrm{~Hz}$ for $10 \mathrm{~min}$ in the presence of normal calcium concentration (13.6 mM) produced a large enhancement of the evokcd EPSPs without alteration of the presynaptic response during the tetanus, and a long-lasting phase (LLP) at $60 \mathrm{~min}$ following the tetanus. Full time course of 5 such experiments is shown in Figure 3B. 4-Aminopyridine, $0.1 \mathrm{mM}$, and TTX, $0.1 \mu \mathrm{M}$, were present in the perfusate for the whole duration of the experiment. 

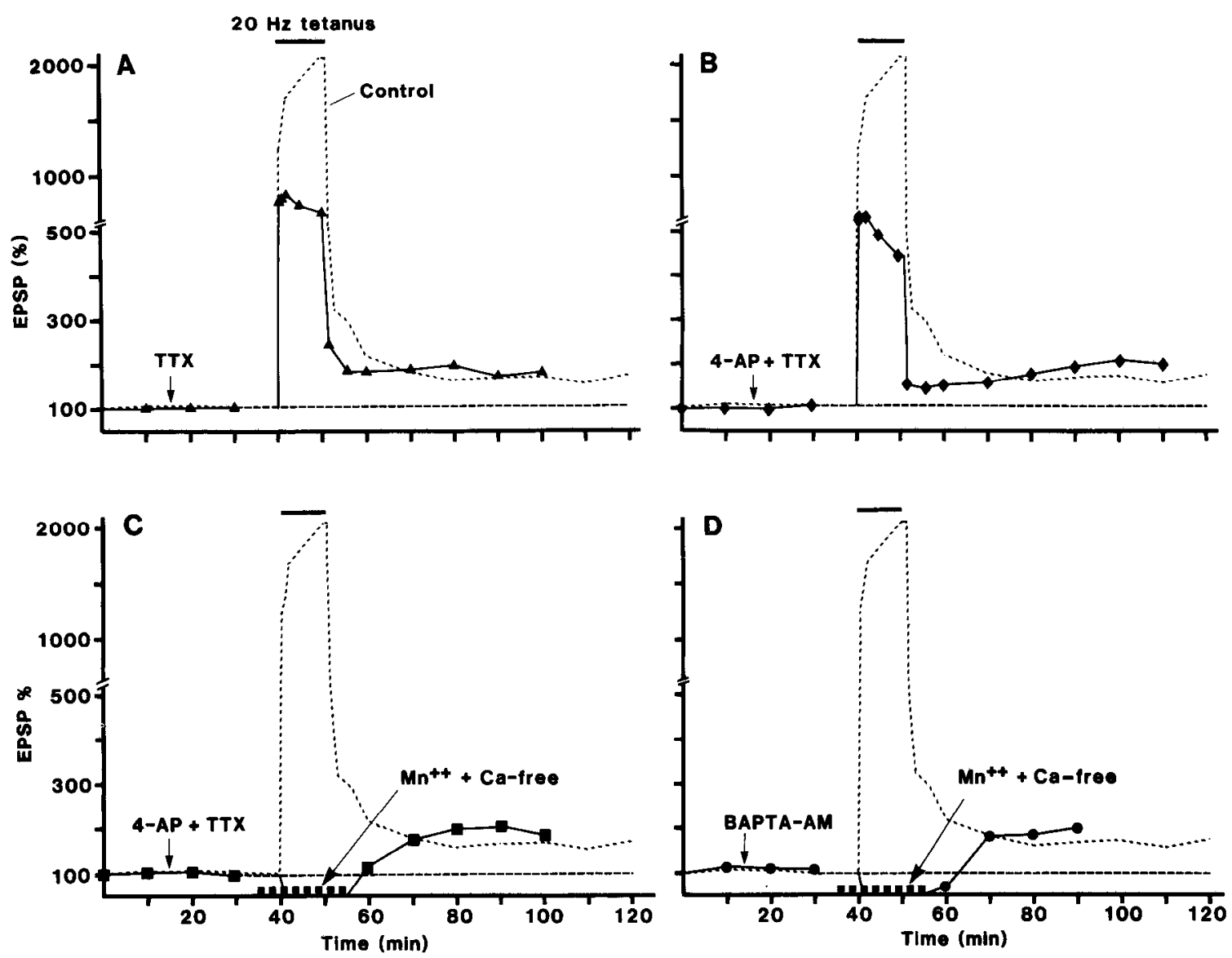

Figure 3. Ionic dissection of LTF. A, Time course of LTF produced by depolarizing pulses in the presence of TTX, i.e., when influx of sodium ions into the terminals was blocked $(n=5)$. Tetanus at $20 \mathrm{~Hz}$ produced a rapid rise of the amplitude of EPSPs (solid line) followed by LLP. Characteristically, the gradual rise of EPSP amplitude during the tetanus and the posttetanic facilitation were absent. $B$, The same in the presence of TTX and 4-aminopyridine $(n=5)$. The effect was very similar, indicating the lack of influence of blocking early K channels on LTF. $C$, Removal of calcium from the perfusate for the duration of tetanic stimulation and its replacement by $6 \mathrm{~mm}$ manganese chloride entirely abolished synaptic transmission but had no effects on LLP following stimulation $(n=8) . D$, Intracellular calcium buffer BAPTA-AM was added to the preparations prior to the experiments in order to reduce the concentration of calcium inside the terminals. In this case the pretest period represents amplitude of EPSPs already depressed by BAPTA. There was still a full expression of LLP even though the tetanus was applied in calcium-free conditions and any release of calcium from intracellular stores was buffered by BAPTA $(n=4)$. The time course of LTF in the presence of normal calcium and sodium concentrations is shown by the broken lines in $A-D$.

upon modification of $\mathrm{K}$ channels (Klein et al., 1982; Alkon, 1984). Measurements were obtained from preparations in which $0.1 \mathrm{~mm}$ 4-aminopyridine (4-AP) was added to the perfusate in addition to TTX. We argued that if changes in potassium conductance were contributing to LTF, pretreatment with the potassium channel blocker 4-AP should reduce the magnitude of LTF. This, however, was not the case (Fig. $3 B$ ). Therefore, it appears that the mechanism responsible for LTF does not involve modification of 4-AP-sensitive $\mathrm{K}$ channels.

In some experiments a component of voltage-dependent potassium conductance appeared to remain after the 4-AP treatment. In Figure $2 B$, for example, the presynaptic voltage pulses show a decrease before the termination of the current pulses. However, there was no evidence that this 4-AP-insensitive conductance changes during LTF. These findings are in accordance with our previous work in which we found no evidence for modification of $\mathrm{K}$ channels in the nerve terminals during LTF evoked by action potentials (Wojtowicz and Atwood, 1986).

Calcium involvement in LTF was explored by exposing the preparation to solutions from which calcium ions were with- drawn and to which the calcium channel blocker manganese (6 mM) was added. The $\mathrm{Ca}^{2+}$-free solution was substituted immediately prior to a period of tetanic stimulation. Synaptic transmission was completely blocked during $20 \mathrm{~Hz}$ stimulation; however, upon restoration of the normal calcium concentration, LLP was present (Fig. 3C). Control experiments showed that the perfusion of preparations $(n=5)$ with calcium-free solutions in the presence of $6 \mathrm{~mm}$ manganese for $20 \mathrm{~min}$ did not produce LLP by blocking the buffering or extrusion of calcium from the terminals. Furthermore there was no noticeable increase of the frequency of spontaneous quantal release after perfusions with manganese. Thus, it is certain that manganese acted by blocking the influx of calcium ions into the terminals but had no important side effects and that repetitive depolarization is a rcquirement for LLP. Similar results were obtained when magnesium (13-30 $\mathrm{mm}$ ) was used as a calcium substitute. However, typically for this preparation, the addition of magnesium did not block synaptic transmission completely so during the tetanic stimulation at $20 \mathrm{~Hz}$ there remained a level of transmitter release equivalent to about $30 \%$ of that evoked in the control 


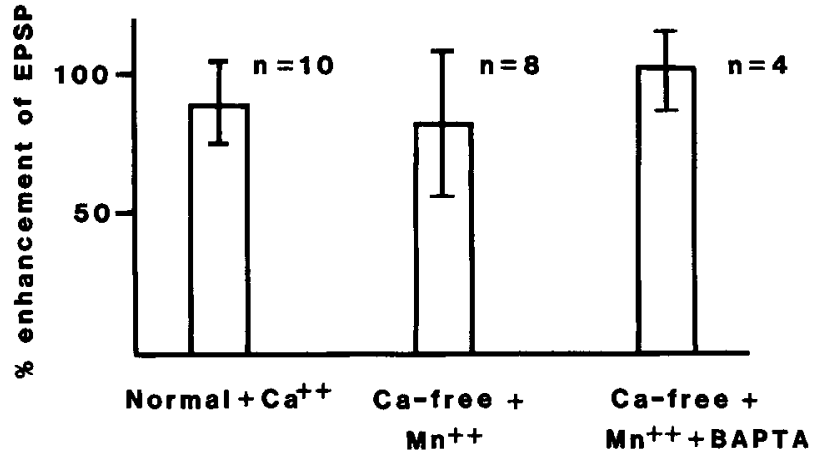

Figure 4. Comparison of the magnitude of LLP at $40 \mathrm{~min}$ following its induction in the presence of calcium ions and during the blockade of their influx into the terminals. The control values were taken from the experiments of the type illustrated in Figure 3, $A, B$, while the others were taken from experiments of the type illustrated in Figure $3, C, D$, respectively. The graph shows that calcium has no effect on the magnitude of LLP. The exact values of the EPSP enhancements were $89.3 \%$ \pm 15.3 (SE) in normal calcium concentration, $82.2 \% \pm 26$ in Ca-free + manganese and $102 \% \pm 15$ in Ca-free + manganese + BAPTA. SEs are indicated.

period at $5 \mathrm{~Hz}$. These results confirm earlier work by Swenarchuk and Atwood (1975) in which it was shown that LTF can be induced by action potentials in Ca-free solutions.

The effects of $\mathrm{NiCl}_{2}$ (up to $1 \mathrm{~mm}$ ) and $\mathrm{CoCl}_{2}$ (up to $0.2 \mathrm{~mm}$ ) on LLP were also tested. These putative calcium channel blockers did not block LLP either. However, the results proved inconclusive since these agents did not block synaptic transmission during the $20 \mathrm{~Hz}$ tetanus completely and in addition produced marked asynchronous release of neurotransmitter.

Further tests were conducted to determine whether buffers for intracellular calcium could modify LTF. BAPTA-AM, one of the recently developed intracellular calcium buffers (Tsien, 1980), was used previously by Charlton and Iwanchyshyn (1986) to suppress the level of intracellular calcium and, consequently, the synaptic transmission at the crayfish neuromuscular junction. In the present study, preparations were pretreated for 1 hr with $2.5 \mu \mathrm{M}$ BAPTA-AM; this reduced synaptic transmission to $44.7 \%$ of normal ( $\mathrm{SE}=6.2 \%, n=6$ ), presumably by binding some of the calcium inside the terminals. Four of these preparations were subsequently stimulated in calcium-free medium with manganese and an undiminished long-lasting enhancement of synaptic transmission ensued (Fig. 3D). The remaining 2 preparations served as controls for possible long-term effects of BAPTA. The result shows that the possible release of calcium from intracellular stores is not likely to cause LLP.

Figure 4 summarizes the data obtained in a number of experiments of the type illustrated in Figure 3. The essential findings are clear. The magnitude of LLP measured $30 \mathrm{~min}$ after the tetanic induction is, as far as we can determine, independent of Ca entry or of an increase in intracellular Ca. Furthermore, since all of these experiments were performed while the influx of Na was blocked with TTX, a role for this latter ion in inducing LTF can also be ruled out.

Another set of experiments was performed to show whether entry of $\mathrm{Ca}^{2+}$ into the nerve terminal could occur without induction of the LLP. We stimulated the terminals at $5 \mathrm{~Hz}$ instead of $20 \mathrm{~Hz}$ in a solution of normal calcium concentration (Fig. 5). Under such conditions, a substantial influx of calcium into the terminals occurred, as evidenced by EPSPs during stimu-

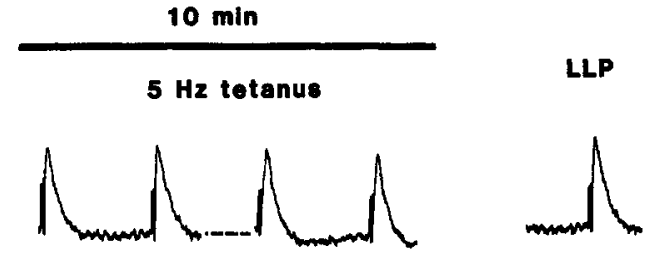

$0.5 \mathrm{mV}$

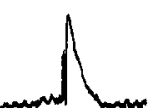

$20 \mathrm{~Hz}$ tetanus + Ca-free + $\mathrm{Mn}^{++}$
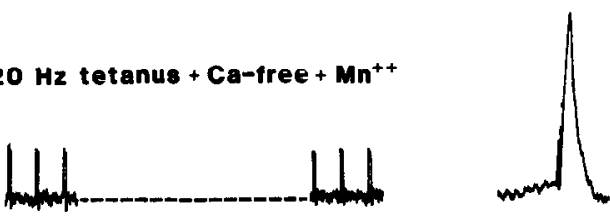

Figure 5. Frequency dependence of LTF. Upon blockade of sodium and potassium currents with TTX and 4-AP, the preparation was stimulated with local depolarizing pulses. Stimulation at $5 \mathrm{~Hz}$ for a period of $10 \mathrm{~min}$ in the presence of normal extracellular $\mathrm{Ca}^{2+}$ had no effect on EPSPs; they remained constant in size during and after the $10 \mathrm{~min}$ period of stimulation. In contrast, stimulation of $20 \mathrm{~Hz}$ during suppression of calcium entry into the synaptic terminals produced clear longlasting enhancement of synaptic transmission (LLP). The result indicates that calcium entry is not a major factor in the induction of LLP and that even when Ca entry occurs, the frequency of depolarization required to induce LLP must exceed $5-10 \mathrm{~Hz}$.

lation. Moreover, the EPSPs were facilitated by about 8 -fold with respect to stimulation at $1 \mathrm{~Hz}$, indicating accumulation of intracellular calcium, but LLP was not produced. In contrast, stimulation at $20 \mathrm{~Hz}$ in Ca-free, Mn-containing solution failed to produce any EPSPs during stimulation, suggesting little or no elevation of intracellular $\mathrm{Ca}^{2+}$, and yet LLP was fully expressed after stimulation when $\mathrm{Ca}^{2+}$ was restored. We conclude that the more frequent depolarizations, rather than $\mathrm{Ca}^{2+}$ entry, are responsible for inducing this phase of LTF.

Finally, in a series of experiments we studicd the induction of LLP in Ca-free solutions with $1 \mathrm{~mm}$ EGTA added to buffer calcium in the extracellular medium. In such conditions a complete block of EPSPs occurred during $20 \mathrm{~Hz}$ stimulation (Fig. 6) and LLP was blocked. The average effect on the EPSP amplitude was $1 \%$ depression (range, -21 to +31 ; $\mathrm{SD}=26.5 ; n$ $=4$ ). Although the result would suggest the requirement for calcium in the induction of LLP, other explanations are possible. In particular, it can be noted in Figure 6 that EGTA caused approximately $10 \mathrm{mV}$ depolarization of presynaptic membrane. Such depolarization did not occur in other experiments where calcium influx was blocked by manganese and may therefore have been the result of membrane destabilization by EGTA. It is possible that this destabilization inactivated a mechanism responsible for induction of LLP. Alternatively, EGTA may have reduced the length constant of the terminal and prevented the depolarization from reaching the terminal during tetanic stimulation.

\section{Characteristics of transmitter release before and after induction of $L L P$}

In 7 preparations transfer curves (see Materials and Methods) were constructed; these indicated that tetanic stimulation produces an increase in the sensitivity of the terminals to a wide range of depolarizations and not only to the ones used for in- 
Presynaptic
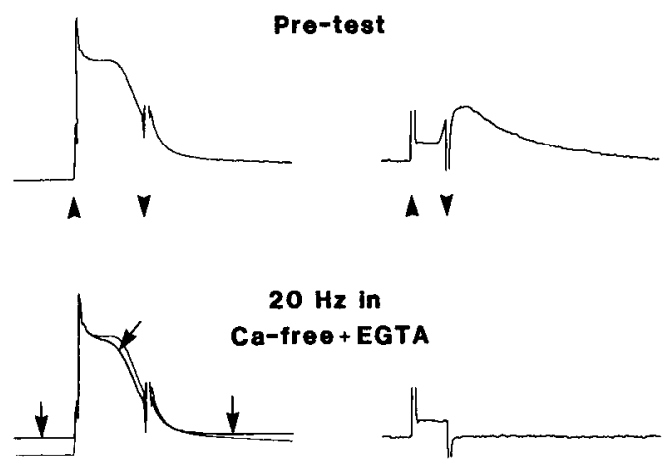

LLP

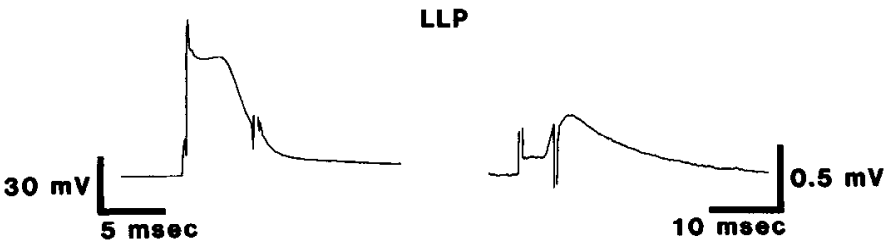

Figure 6. Effects of Ca-free + EGTA solution on the establishment of LLP. Presynaptic depolarizing pulses in axonal terminal are shown on the left and the evoked EPSPs on the right. Pretest records were obtained by applying $60 \mathrm{nA}, 5 \mathrm{msec}$ current pulses at the frequency of $5 \mathrm{~Hz}(100$ averaged pulses are shown). During the tetanic phase (middle records), the preparation was perfused by $\mathrm{Ca}$-free solution with the addition of $1 \mathrm{~mm}$ EGTA and $13.5 \mathrm{~mm} \mathrm{MgCl}_{2}$. The effects of this treatment were a blockade of EPSP and depolarization of presynaptic membrane by about $10 \mathrm{mV}$. In order to demonstrate the latter effect, the tetanic record was superimposed on the pretest record. Arrows point at the tetanic record. It is evident that $\mathrm{Ca}$-free + EGTA solution also reduced the amplitude of the pulse slightly. As discussed in the text, these effects can be accounted for by the blockade of transmitter release and an additional destabilizing effect on the membrane, probably due to removal of the calcium screening charge from the membrane. The bottom records show the lack of LLP. A clear LLP in the same preparation is shown in Figure $2 B$.

duction of the effect (Fig. 1C). When plotted on a logarithmic scale the initial segment of the transfer curves appeared linear with a slope of near 4.0 (range, 3.5-7). The similarity of these values to the values of cooperativity between calcium and a release mechanism for neurotransmitter obtained by others ( $\mathrm{Au}$ gustine and Charlton, 1986; Parnas et al., 1986) may be fortuitous because the present method does not allow a good estimate of intracellular calcium concentration. Pending further investigation, our data should be interpreted simply as a method of measuring the functional status of the synaptic terminals.

In 3 experiments we measured the degree of short-term facilitation before and after the establishment of LLP. We found that the short-term facilitation, defined as the ratio of the amplitude of evoked EPSPs at the frequency of $5 \mathrm{~Hz}$ to that at the frequency of $1 \mathrm{~Hz}$ was nol significanlly affected by LLP: $7.8 \pm$ 4.1 (SD) before and $7.2 \pm 3.2(\mathrm{SD})$ after LLP $(n=3)$. Similarly, there was no noticeable effect of LLP on the rate of asynchronous quantal release measured during low-frequency stimulation (1$5 \mathrm{~Hz}$ ).

We also analyzed quantal fluctuations of pulse-evoked EPSPs using methods employed previously (Wojtowicz and Atwood, 1986). The mean amplitude of miniature EPSPs was not changed by LTF. It averaged $0.24 \mathrm{mV}(\mathrm{SD} \pm 0.11$ ) before and $0.23 \mathrm{mV}$ $(\mathrm{SD} \pm 0.09)$ after the tetanus $(n=7)$. However, the mean quantal content was increased in proportion to the increase of the averaged evoked EPSPs. Thus, in 3 representative experiments analyzed in detail the quantal content of postsynaptic responses increased from $2.2 \pm 0.6(\mathrm{SD})$ to $3.6 \pm 0.64$, representing a $64 \%$ enhancement in transmitter release. In the same preparations the amplitude of the averaged EPSPs, such as shown in Figure $2 B$, was increased by $70 \%$. These results are in agreement with a previous study in which we analyzed quantal release by action potentials during LTF (Wojtowicz and Atwood, 1986). The results indicate that pulse-evoked and action potentialevoked LLP employ the same presynaptic mechanisms.

\section{Discussion}

LLP resembles in its magnitude and its duration the long-term potentiation phenomenon of the mammalian brain. The mechanisms responsible for the induction of long-term potentiation are still under investigation, with the bulk of evidence pointing to the role of calcium influx (Turner et al., 1982; Lynch and Baudry, 1984) into the postsynaptic neurons via voltage-dependent $N$-methyl-D-aspartate channels (Wigstrom and Gustafsson, 1985; Wigstrom et al., 1986) and other evidence arguing against a role for calcium in certain of the neural circuits which show long-term potentiation (May et al., 1987). The locus (or loci) of long-term potentiation is not known with certainty, although the experimental evidence obtained thus far suggests that it is at least partly presynaptic (Malenka et al., 1986; Errington, et al., 1987).

The present study indicates a new mechanism that operates to produce LTF in the crayfish nerve terminals. Specifically, we found that the induction of LLP can be produced by repeated depolarizations of the nerve terminals under conditions in which $\mathrm{Na}$ and $\mathrm{Ca}$ entry are not occurring. Thus, the underlying mechanism for LLP is voltage dependent, yet influx and intracellular build-up of calcium does not appear to play a role. This conclusion is based primarily on experiments showing that a clear LLP was induced after the manganese blockade of synaptic transmission and $20 \mathrm{~Hz}$ stimulation. Stimulation at $5 \mathrm{~Hz}$ did not produce LLP even though a significant calcium influx and accumulation occurred (see Figs. $3 D, 4,5$ ). We also confirmed that LLP is homosynaptic, i.e., that it can be produced by repetitive stimulation of a single, identified axon. Finally, the locus for LLP appears to be in the presynaptic terminal and not in the postsynaptic cells. This conclusion is based on the finding of the constancy of quantal size, an increase in quantal output, and a shift in the transfer curve of the nerve terminal to applied depolarization. It remains to be determined if mechanisms similar to those described here also operate in the vertebrate nervous system.

We have previously observed (Wojtowicz and Atwood, 1985b) and confirm here that blockade of LLP occurs in calcium-free medium if the calcium buffer EGTA is added to the external medium. In these experiments the block of LLP may have been due to a reversed gradient for $\mathrm{Ca}^{2+}$ across the nerve terminal membrane (Rahamimoff et al., 1980), which resulted in a lowering of $\mathrm{Ca}^{2+}$ inside the terminals below the normal resting level. Such an action of EGTA may have a deleterious effect on the presynaptic membrane and biochemical processes required for the induction of LLP. Alternatively, EGTA may have acted by directly destabilizing the presynaptic membrane and thus inhibiting LLP. A destabilization of the membrane was, in fact, observed, and on several occasions it resulted in a loss of seal around the presynaptic microelectrode and subsequent termi- 
nation of the experiments. Whatever the effects of EGTA were, our former proposal that LLP is calcium dependent (Wojtowicz and Atwood, 1985b; Atwood and Wojtowicz, 1986) can no longer be sustained in view of the overwhelming evidence to the contrary from the experiments demonstrated in Figure 3 (effects of mangancse) and Figurc 5. These latter expcriments showed that even relatively large calcium influx occurring during $5 \mathrm{~Hz}$ stimulation is insufficient for the induction of LLP. Furthermore, BAPTA had no visible deleterious effects on the membrane and did not block LLP even when applied in conjunction with the calcium-free medium. An analogous observation was made by Best and Bolton (1986), who found that depolarization of smooth muscle cells may trigger the hydrolysis of inositol phospholipids independently of $\mathrm{Ca}$ influx, yet when $\mathrm{Ca}$ in the extracellular space is buffered with EGTA the hydrolysis is blocked.

Our experiments with BAPTA argue against a role of intracellular calcium stores in the induction of LLP. Although an accurate estimate of the concentration of intracellular calcium was not possible in this study, BAPTA had a considerable buffering capacity and reduced evoked EPSPs at $5 \mathrm{~Hz}$ by about $50 \%$. However, this buffering capacity had no influence on the magnitude of LLP induced in the Ca-free medium (Figs. 3, 4).

The question remains as to how presynaptic depolarization elicits LLP. It is difficult to completely rule out a possible role for ions other than $\mathrm{Ca}$ and $\mathrm{Na}$ since some ionic fluxes such as chloride influx or 4-AP-insensitive $\mathrm{K}$ efflux must accompany the presynaptic depolarization. Roles for chloride or potassium as messenger ions are unlikely because chloride freely equilibrates across the nerve terminal membrane (Takeuchi and Takeuchi, 1966), and the intracellular concentration of potassium is relatively high. A long-lasting accumulation of calcium ions seems to be ruled out by the lack of elevation of the rate of asynchronous quantal release. A simpler and more likely explanation in our view is that the long-lasting component of LTF is induced by a mechanism that depends on the electrical field. Perhaps a voltage-sensitive "synaptic switch" undergoes a conformational change in the presynaptic membrane as a result of repeated depolarizations and leads to enhanced transmitter release.

A recently advanced voltage-calcium hypothesis of transmitter release (Dudel et al., 1983) provides one possible mechanism via which voltage may alter the synaptic release process. According to this hypothesis, the release is dependent not only on the presence of calcium ions but also on an initial voltagedependent step which may be altered during repetitive depolarizations. However, preliminary observations (not illustrated) show that LLP cannot be blocked by hyperpolarizing pulses applied in sequence with the depolarizing ones. In such experiments $(n=5)$, the standard depolarizing pulses were followed by hyperpolarizing ones of equal amplitude but with $5-10 \mathrm{msec}$ delay. Bouts of $20 \mathrm{~Hz}$ stimulations with such twin pulses produced clear LLP in most cases. This result leads us to propose an alternative mechanism in which the depolarizations may trigger release of a molecule from the presynaptic membrane, perhaps a component of a second-messenger system, which in turn signals a functional change in the status of synaptic release sites. We think that this is a new and exciting possibility open for future research.

Note added in proof: A recent paper by Wojtowicz et al. (1988) provides more experimental evidence for arguments presented in this paper.

\section{References}

Alkon, D. L. (1984) Calcium-mediated reduction of ionic currents: A biophysical memory trace. Science 226:1037-1045.

Atwood, H. L. (1976) Organization and synaptic physiology of crustacean neuromuscular systems. Prog. Neurobiol. 7: 291-391.

Atwood, H. L., and J. M. Wojtowicz (1986) Short-term and long-term plasticity and physiological differentiation of crustacean motor synapses. Int. Rev. Neurobiol. 28: 275-362.

Atwood, H. L., L. E. Swenarchuk, and C. R. Gruenwald (1975) Longterm synaptic facilitation during sodium accumulation in nerve terminals. Brain Res. 100: 198-204.

Augustine, G. J., and M. P. Charlton (1986) Calcium dependence of presynaptic calcium current and post-synaptic response at the squid giant synapse. J. Physiol. (Lond.) 381: 619-640.

Augustine, G. J., M. P. Charlton, and S. J. Smith (1987) Calcium action in synaptic transmitter release. Annu. Rev. Neurosci. 10:633693.

Best, L., and T. B. Bolton (1986) Depolarization of guinea-pig visceral smooth muscle causes hydrolysis of inositol phospholipids. Naunyn Schmiedebergs Arch. Pharmacol. 333: 78-82.

Charlton, M. P., and G. J. Iwanchyshyn (1986) Exogenous calcium buffer reduces synaptic transmitter release and facilitation. Soc. Neurosci. Abstr. 12:817.

Dudel, J. (1984) Control of quantal transmitter release at frog's motor nerve terminals. I. Dependence on amplitude and duration of depolarization. Pfluegers Arch. 402: 225-234.

Dudel, J., I Parnas, and H. Parnas (1983) Neurotransmitter release and its facilitation in crayfish muscle. VI. Release determined by both intracellular calcium concentration and depolarization of the nerve terminal. Pfluegers Arch. 399: 1-10.

Errington, M. L., M. A. Lynch, and T. V. P. Rliss (1987) Long-term potentiation in the dentate gyrus: Induction and increased glutamate release are blocked by $D(-)$ aminophosphovalerate. Neuroscience 20 : $279-284$.

Erulkar, S. D. (1983) The modulation of neurotransmitter release at synaptic junctions. Rev. Physiol. Biochem. Pharmacol. 98: 63-175.

Katz, B., and R. Miledi (1967) The release of acetylcholine from nerve endings by graded electric pulses. Proc. R. Soc. London [Biol.] 167: 23-28.

Katz, B., and R. Miledi (1968) The role of calcium in neuromuscular facilitation. J. Physiol. (Lond.) 195: 481-492.

Klein, M. J. Camardo, and E. R. Kandel (1982) Serotonin modulates a specific potassium current in the sensory neurons that show presynaptic facilitation in Aplysia. Proc. Natl. Acad. Sci. USA 79: 57135717

Lnenicka, G. A., and H. L. Atwood (1985) Long-term facilitation and long-term adaptation at synapses of a crayfish phasic motoneuron. $\mathrm{J}$. Neurobiol. 16: 97-110.

Lynch, G., and M. Baudry (1984) The biochemistry of memory: A new and specific hypothesis. Science 224: 1057-1063.

Lynch, G., J. Larson, S. Kelso, G. Barrionuevo, and F. Schottler (1983) Intracellular injections of EGTA block induction of hippocampal longterm potentiation. Nature 305: 719-721.

Malenka, R. C., D. V. Madison, and R. A. Nicoll (1986) Potentiation of synaptic transmission in the hippocampus by phorbol esters. Nature 321: $175-177$.

May, P. B. Y., J. W. Goh, and B. R. Sastry (1987) Induction of hippocampal long-term potentiation in the absence of extracellular Ca. Synapase 1: 273-278.

Parnas, H., I. Parnas, and L. Segel (1986) A new method for determining cooperativity in neurotransmitter release. J. Theor. Biol. 119: 481-499.

Parnas, I., and H. Parnas (1986) Calcium is essential but insufficient for neurotransmitter release: The calcium-voltage hypothesis. J. Physiol. (Paris) 81: 289-305.

Rahamimoff, R., A. Lev-Tov, and H. Meiri (1980) Primary and secondary regulation of quantal transmitter release: Calcium and sodium. J. Exp. Biol. 89: 5-18.

Schwartz, E. A. (1987) Depolarization without calcium can release $\gamma$-aminobutyric acid from a retinal neuron. Science $238: 350-355$.

Sherman, R. B., and H. L. Atwood (1971) Synaptic facilitation: Longterm neuromuscular facilitation in crustaceans. Science $171: 1248-$ 1250 .

Swenarchuk, L. E., and H. L. Atwood (1975) Long-term facilitation with minimal calcium entry. Brain Res. 100: 205-208.

Takcuchi, A., and N. Takeuchi (1966) On the permeability of the 
presynaptic terminal of the crayfish neuromuscular junction during synaptic inhibition and the action of $\gamma$-aminobutyric acid. J. Physiol. (Lond.) 183: 433-449.

Tsien, R. Y. (1980) New calcium indicators and buffers with high selectivity against magnesium and protons: Design, synthesis, and properties of prototype structures. Biochemistry 19: 2396-2404.

Tsien, R. Y. (1981) A non-disruptive technique for loading calcium buffers and indicators into cells. Nature 290: 527-528.

Turner, R. W., K. G. Baimbridge, and J. J. Miller (1982) Calciuminduced long-term potentiation in the hippocampus. Neuroscience 7 : 1411-1416.

Wigstrom, H., and B. Gustafsson (1985) On long-lasting potentiation in the hippocampus: A proposed mechanism for its dependence on coincident pre- and postsynaptic activity. Acta Physiol. Scand. 123: 519-522.

Wigstrom, H., B. Gustafsson, Y. Y. Huang, and W. C. Abraham (1986) Hippocampal long-term potentiation is induced by pairing single afferent volleys with intracellularly injected depolarizing current pulses. Acta Physiol. Scand. 126: 317-319.

Wojtowicz, J. M., and H. L. Atwood (1984) Presynaptic membrane potential and transmitter release at the crayfish neuromuscular junction. J. Neurophysiol. 52: 99-113.

Wojtowicz, J. M., and H. L. Atwood (1985a) Correlation of presynaptic and postsynaptic events during establishment of long term facilitation at the crayfish neuromuscular junction. J. Neurophysiol. 54: 220-230.

Wojtowicz, J. M., and H. L. Atwood (1985b) Calcium-dependent longterm enhancement of synaptic transmission without action potentials in axonal terminals at the crayfish neuromuscular junction. Soc. Neurosci. Abstr. 11: 965.

Wojtowicz, J. M., and H. L. Atwood (1986) Long-term facilitation alters transmitter releasing properties at the crayfish neuromuscular junction. J. Neurophysiol. 55: 484-498.

Wojtowicz, J. M., H. Parnas, I. Parnas, and H. L. Atwood (1988) Long-term facilitation of synaptic transmission demonstrated with macro-patch recording at the crayfish neuromuscular junction. Neurosci. Lett. 90: 152-158.

Zucker, R. S., and L. Lando (1986) Mechanism of transmitter release: Voltage hypothesis and calcium hypothesis. Science 231: 574-579. 